\title{
Influence of Surface Force on Motion of An Electrostatic Microstructure
}

\author{
Member Shigeki Tsuchitani (Wakayama University) \\ Non-Member Reizo Kaneko \\ (Wakayama University) \\ Member Seiko Suzuki \\ (Hitachi Ltd.)
}

In an electrostatic microstructure with a movable electrode supported by a cantilever beam and counter electrodes, voltage dependency of displacement of the movable electrode is studied by taking surface force into consideration. The displacement change is analyzed theoretically with the energy model which determines the stable displacement state of the movable electrode from the minimum energy state of the total energy of elastic energy, electrostatic energy and adhesion energy between two surfaces. The relation between the surface force at contact parts of the movable electrode with the counter one and a hysteresis voltage of capacitance (between the both electrodes)-voltage characteristics (C-V characteristics) was investigated and a magnitude range of the surface force in which stiction of the movable electrode occurs is derived. In an experiment which simulated electrical contacts of the electrostatic microstructure with charged objects, the surface force increased with increase of the charge amount of the object. In measurements of $\mathrm{C}-\mathrm{V}$ characteristics in positive and negative voltage regions, the $\mathrm{C}-\mathrm{V}$ characteristics shifted to the positive voltage region and the shift was larger in a device with larger surface oxide layer of the movable electrode. This voltage shift is thought to be caused by a positive electrical charge remaining in the oxide layer.

Keywords: Microstructure, Electrostatic force, Surface force, Energy model, Electrification, Oxide charges

\section{INTRODUCTION}

In electrostatic microstructures such as micro scanners, micro relays and servo accelerometers, mechanical response of a movable electrode to an electrostatic applied force is essential for their operation. Application of the electrostatic force to the movable electrode is also carried out in self-diagnosises of the electrostatic microstructures when they are used in systems which demand high reliabilities [1]. The electrostatic force can be easily applied to the movable electrode only by forming a counter fixed electrode, and the magnitude of the force is easily changed by changing the voltage between the both electrodes. Therefore, in these devices, it is important to know precisely the behavior of the movable electrode under the application of the electrostatic force between the both electrodes.

On the other hand, surface forces between two counter surfaces have also large influences on the operations of the microstructures [2-6].

In this paper, we have investigated a mechanical response of a movable electrode of an electrostatic microstructure under the electrostatic force applied between the movable and the fixed electrodes. Effects of the surface force to the response characteristics are taken into consideration. Voltage dependencies of the motion of the

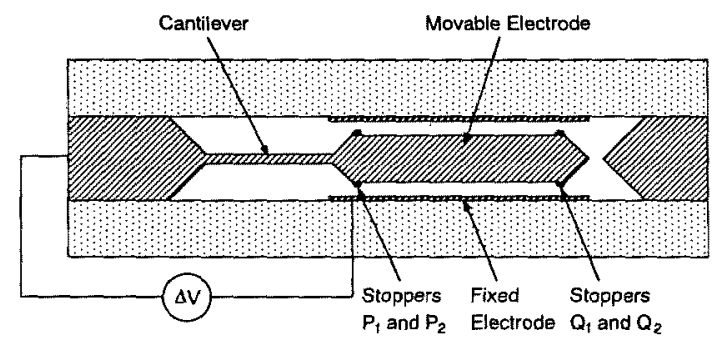

Figure 1: Structure of the electrostatic microstructure studied

movable electrode is analyzed theoretically with the energy model. Some experimental results of the response characteristics, which show the effects of the surface force, are described.

\section{THEORETICAL ANALYSIS OF CAPACITANCE -VOLTAGE CHARACTERISTICS}

Figure 1 shows a cross section of the electrostatic microstructure studied. It has a silicon movable electrode, a silicon cantilever beam to support the movable electrode and counter fixed electrodes formed by a thin metal film (Mo). To avoid a direct electrical contact of the movable electrode with the fixed one, small stoppers $P_{1}, P_{2}, Q_{1}$ and 
Table 1: Various dimensions of the electrostatic microstructure

\begin{tabular}{|l|l|r|}
\hline \multirow{3}{*}{ Cantilever } & Length $\left(\mathrm{L}_{1}\right)$ & 800 \\
\cline { 2 - 3 } & Width $\left(\mathrm{W}_{1}\right)$ & 250 \\
\cline { 2 - 3 } & Thickness $\left(\mathrm{T}_{1}\right)$ & 16 \\
\hline \multirow{2}{*}{$\begin{array}{l}\text { Movable } \\
\text { electrode }\end{array}$} & Length $\left(\mathrm{L}_{2}\right)$ & 1300 \\
\cline { 2 - 3 } & Width $\left(\mathrm{W}_{2}\right)$ & 1800 \\
\cline { 2 - 3 } & Thickness $\left(\mathrm{T}_{2}\right)$ & 210 \\
\hline Stopper & Height $(\mathrm{H})$ & 0.8 \\
\hline Gap width $\left(\mathrm{D}_{0}\right)$ & 3.0 \\
\hline
\end{tabular}

(Unit: $\mu \mathrm{m})$

$\mathrm{Q}_{2}$ of an insulator $\left(\mathrm{SiO}_{2}\right)$ were formed at four corners of each counter surface of the movable electrode. Table 1 shows device dimensions used in the calculation.

When the deformation of the movable electrode and the twist of the cantilever beam are small, the displacement state of the movable electrode is determined by the displacement $Y_{p}$ of the stoppers $P_{1}$ and $P_{2}$ and the displacement $Y_{q}$ of the stoppers $Q_{1}$ and $Q_{2}$. The behavior of the movable electrode for an applied voltage between the both electrodes is determined by the total energy of elastic energy $U_{m}$ stored in the cantilever beam, electrostatic energy $U_{e}$ of an electric field between the electrodes and adhesion energy $U_{a d}$ between the stoppers and the fixed electrode when they contact each other.

The elastic energy $U_{m}$ is calculated by the next equation [7].

$$
U_{m}=(1 / 2) \int_{0}^{L_{l}}\left(M^{2} / E I\right) d x
$$

Where $E$ is the Young's modulus of the cantilever beam material (single crystal silicon), $I$ is the moment of inertia of the beam section and $\mathrm{L}_{1}$ is the length of the cantilever beam. $M$ is the bending moment at the $\mathrm{x}$-section (the sign is positive when the upper layer of the beam is compressed). The bending moment $\mathrm{M}$ is determined uniquely for a given displacement state $\left(Y_{p}, Y_{q}\right)$ and expressed as follows.

$$
M=A x+B
$$

Where $\mathrm{A}$ and $\mathrm{B}$ are given by the next equations.

$$
\begin{aligned}
& A=\left(\frac{6 E I}{L_{1}{ }^{2}}\right)\left(\frac{Y_{q}-Y_{p}}{L_{2}}-\frac{2 Y_{p}}{L_{1}}\right) \\
& B=-\left(\frac{2 E I}{L_{1}}\right)\left(\frac{Y_{q}-Y_{p}}{L_{2}}-\frac{3 Y_{p}}{L_{1}}\right)
\end{aligned}
$$

Where $\mathrm{L}_{2}$ is the length of the movable electrode. By substituting eq.(2) into eq.(1), the elastic energy $U_{m}$ is expressed by the displacements $Y_{p}$ and $Y_{q}$ through the parameters $\mathrm{A}$ and $\mathrm{B}$, as shown in eq.(5).

$$
U_{m}=(1 / 6 E I)\left(A^{2} L_{1}^{2}-3 A B L_{1}+3 B^{2}\right) L_{1}
$$

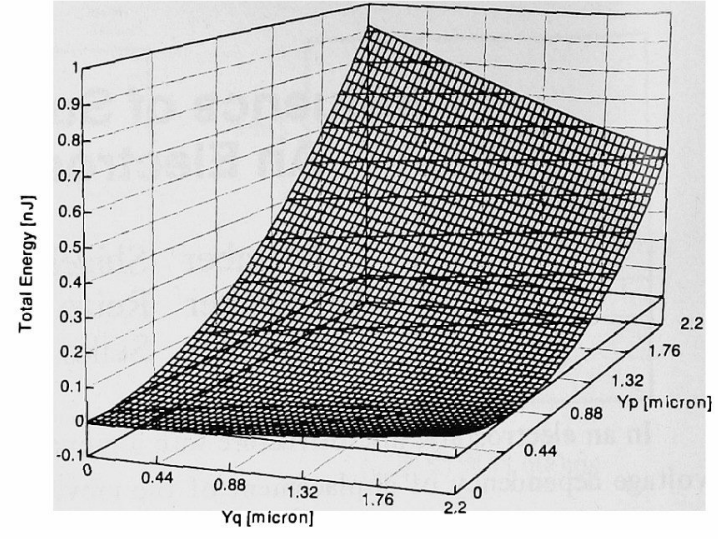

Figure 2: Total energies in various displacement states of the movable electrode at $V=I V$

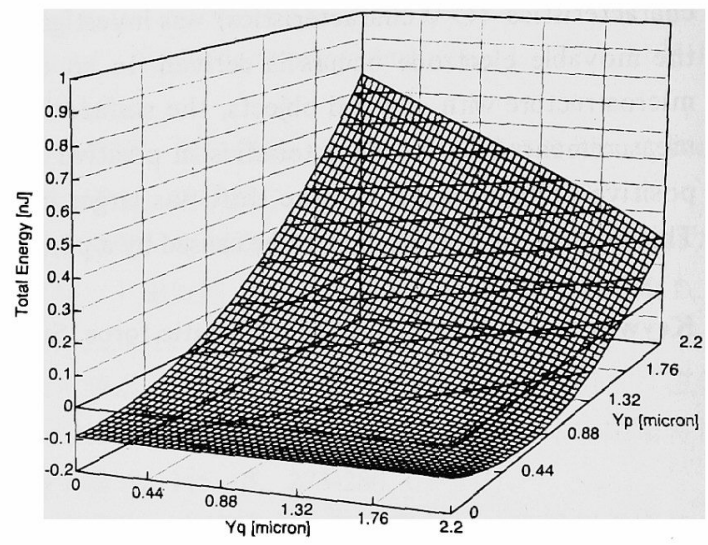

Figure 3: Total energies in various displacement states of the movable electrode at $V=5 \mathrm{~V}$

On the other hand, the electrostatic energy $U_{c}$ is given by eq.(6).

$$
\mathrm{U}_{\mathrm{e}}=-(1 / 2) \mathrm{CV}^{2}
$$

Where $\mathrm{C}$ is a capacitance between the both electrodes and expressed as follows also by using the displacements $\mathrm{Y}_{\mathrm{p}}$ and $\mathrm{Y}_{\mathrm{q}}$.

$$
C=\frac{\varepsilon_{0} S}{Y_{q}-Y_{p}} \log \left(\frac{D_{0}-Y_{p}}{D_{0}-Y_{q}}\right)
$$

Where $\mathrm{S}$ is the counter surface area of the both electrodes, $D_{0}$ is the gap width between them when the displacement of the movable electrode is zero and $\varepsilon_{0}$ is the dielectric constant of vacuum.

As the effect of the attractive surface force, the following adhesion energy $U_{a d}$ was added to the sum of the elastic energy $U_{m}$ and the electrostatic energy $U_{e}$ only in the deformation states in which the movable electrode contacted with the fixed one through the stoppers.

$$
U_{a d}=-F_{a d} Y_{a d}
$$




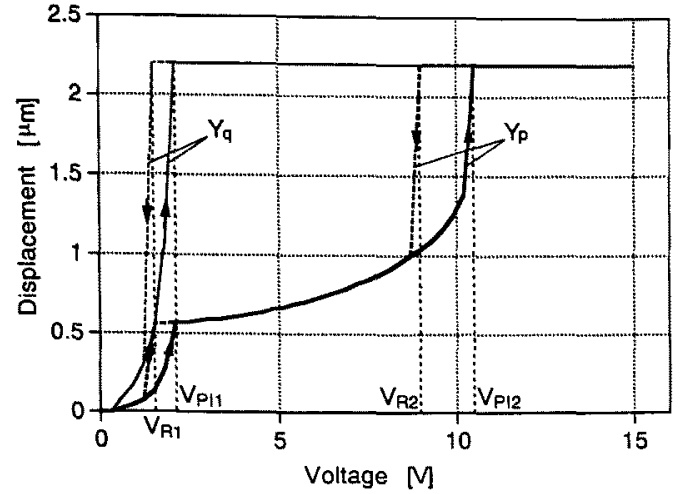

Figure 4: Voltage dependence of the displacements $Y_{n}$ and $Y_{q}$

Where $Y_{a d}$ is the force range of the surface force $F_{a d}$ and was given by the next equation.

$$
\mathrm{Y}_{\mathrm{ad}}=\left(\mathrm{D}_{0}-\mathrm{H}\right) / \mathrm{N}
$$

Where $\mathrm{H}$ is the height of the stoppers and $\mathrm{N}$ is the number of discrete values of the displacement $Y_{p}$ or $Y_{q}$ in the numerical calculation.

Figures 2 and 3 show dependencies of the total energy $U_{\text {tot }}=U_{m}+U_{e}$ on the displacement state $\left(Y_{p}, Y_{q}\right)$ of the movable electrode when the surface force is zero and the applied voltages are $1 \mathrm{~V}$ and $5 \mathrm{~V}$, respectively. The stable displacement state of the movable electrode in each voltage is given by the displacement state $\left(\mathrm{Y}_{\mathrm{p}}, \mathrm{Y}_{q}\right)$ in which the total energy $U_{t o r}$ is minimum. The minimum energy state is $\left(Y_{p}, Y_{q}\right)=(0.04 \mu m, 0.18 \mu m)$ at $V=1 V$ and $\left(Y_{p}, Y_{q}\right)=$ $(0.66 \mu \mathrm{m}, 2.20 \mu \mathrm{m})$ at $\mathrm{V}=5 \mathrm{~V}$.

Figure 4 shows calculated changes of the displacements $Y_{p}$ and $Y_{q}$ in the stable displacement states when the voltage is increased from 0 to $15 \mathrm{~V}$ and decreased from $15 \mathrm{~V}$ to 0 . In the calculation, the surface force which acts in the contact parts of the stoppers and the fixed electrode, is supposed to be zero. The stable displacement states were calculated by the voltage step of $0.3 \mathrm{~V}$.

In the voltage increase process, the displacements $Y_{p}$ and $Y_{4}$ increase suddenly near $V=2.1 \mathrm{~V}$ (the first pull-in voltage $: V_{\mathrm{PII}}$ ) and reach $0.57 \mu \mathrm{m}$ and $2.20 \mu \mathrm{m}$, respectively. This means that only the stoppers $Q_{1}$ and $Q_{2}$ which are formed near the tip of the movable electrode contact with the fixed electrode at this voltage. In the voltage larger than $2.1 \mathrm{~V}$, the displacement $\mathrm{Y}_{\mathrm{q}}$ is constant to $15 \mathrm{~V}$. On the other hand, the displacement $Y_{p}$ increases gradually to $10.2 \mathrm{~V}$ and raises up to $2.20 \mu \mathrm{m}$ at $10.5 \mathrm{~V}$ (the second pull-in voltage $: \mathrm{V}_{\mathrm{P} / 2}$ ). At this voltage, all the stoppers contact with the fixed electrode.

In the voltage decrease process, the displacement $Y_{p}$ falls down at $9.0 \mathrm{~V}$ and $1.5 \mathrm{~V}$ and the displacement $Y_{q}$ at

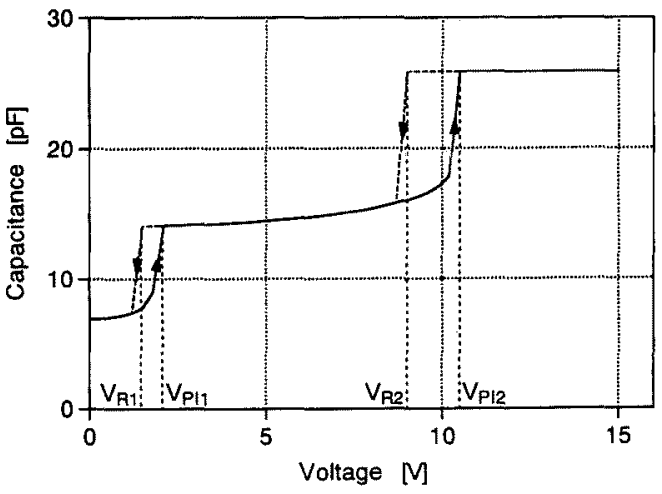

Figure 5: Voltage dependence of the capacitance between the movable and the fixed electrodes

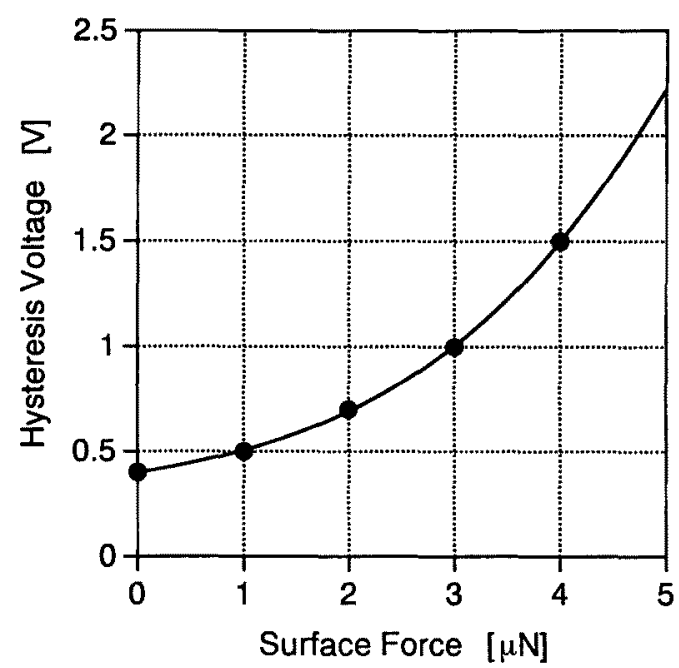

Figure 6: Surface force (per one stopper) dependence of hysteresis voltage $V_{H / T}-V_{k J}$

$1.5 \mathrm{~V}$. That is to say, the stoppers $\mathrm{P}_{1}$ and $\mathrm{P}_{2}$ separate from the fixed electrode first at $9.0 \mathrm{~V}$ (the second release voltage: $V_{R 2}$ ) and then the stoppers $Q_{1}$ and $Q_{2}$ separate at $1.5 \mathrm{~V}$ (the first release voltage: $V_{R 1}$ ).

Figure 5 shows the capacitance change of the stable displacement state calculated by eq.(7) from the displacement changes in Fig. 4. The capacitance change in the voltage increase process and that in the voltage decrease process are different, i.e. there exists a hysteresis. We call the difference between the pull-in voltage and the corresponding release voltage as a hysteresis voltage.

The hysteresis voltage is influenced by the surface force between the stoppers and the fixed electrode. Figure 6 shows the surface force dependence of the hysteresis voltage $\left(V_{P I}-V_{R I}\right)$. The horizontal axis indicates the surface force per one stopper. The hysteresis voltage increases nonlinearly with the increase of the surface force. When 


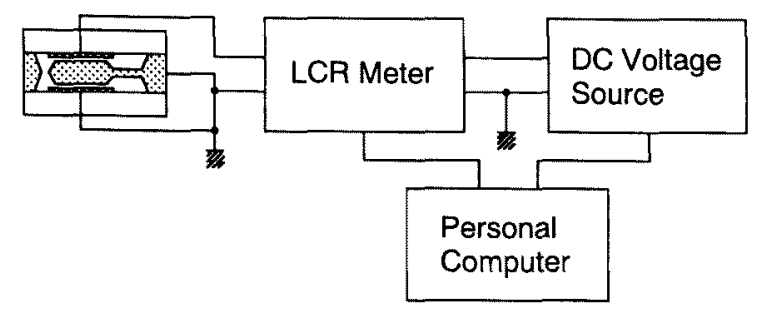

Figure 7: Measurement circuit of the capacitance change

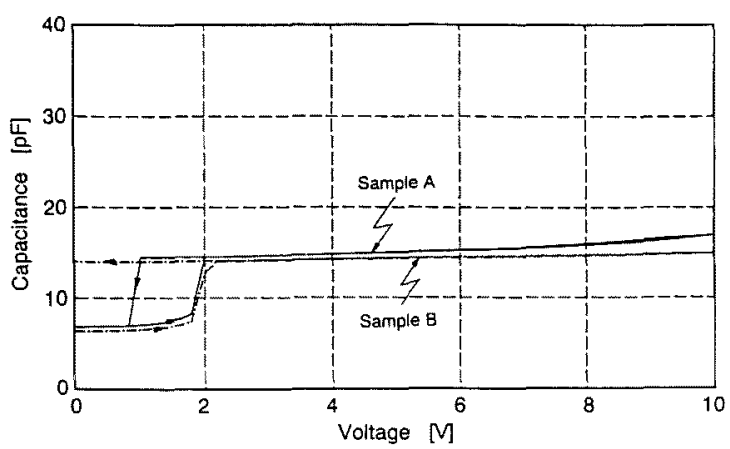

Figure 8: Examples of measured capacitance-voltage characteristics

the surface force is larger than $4.7 \mu \mathrm{N}$, the hysteresis voltage becomes larger than the magnitude of the first pull-in voltage $2.1 \mathrm{~V}$. This means that the movable electrode does not return to the original position when the voltage is reduced to zero, i.e. a stiction between the movable electrode and the fixed one arises.

\section{EXPERIMENTAL RESULTS AND DISCUSSION}

Voltage dependencies of the capacitance between the movable and the fixed electrodes of sample devices were measured by a circuit shown in Figure. 7. LCR meter was used in a measurement condition of $100 \mathrm{kHz}$ and $50 \mathrm{mV}$ (effective voltage), and DC bias voltage which was changed by a step of $0.1 \mathrm{~V}$ or $0.2 \mathrm{~V}$ was added to the measurement $\mathrm{AC}$ voltage. The silicon part of the device was connected to the ground potential level and a positive or a negative voltage was applied to one of the fixed electrodes. The other fixed electrode was also connected to the ground level to avoid an application of the electrostatic force between this electrode and the movable one. The gravitational force perpendicular to the movable electrode was zero.

Figure 8 shows examples of measured capacitancevoltage characteristics ( $\mathrm{C}-\mathrm{V}$ characteristics). The applied voltage was increased from $0 \mathrm{~V}$ to $+10 \mathrm{~V}$ and then was decreased from $+10 \mathrm{~V}$ to $0 \mathrm{~V}$. There existed the hysteresis in

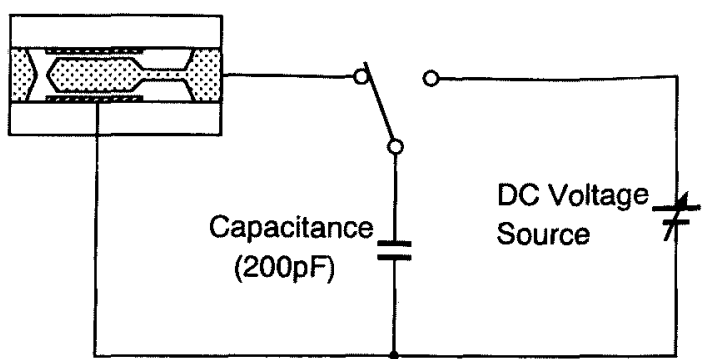

Figure 9: Circuit for simulatting contact of the electrostatic microstructure with charged body

the characteristics of the samples A and $\mathrm{B}$. The capacitance of the sample $B$ didn't return to the original value when the voltage was decreased to zero. As described in the above, this is thought to be caused by the stiction of the movable electrode to the fixed one due to the surface force between the stoppers $Q_{1}$ and $Q_{2}$ and the fixed electrode. The total surface force was estimated at about $6 \mu \mathrm{N}$ in the sample $\mathrm{A}$ and larger than $9.5 \mu \mathrm{N}$ in the sample $\mathrm{B}$ from the relation of Fig. 4 .

It is known that the surface force in the microstructures is influenced by many kinds of factors such as humidity, degree of hydrophilicity of the surface, heat treatment and electrification of an intermediate insulation layer between the movable and the fixed electrodes [4-5]. In the next, we will describe influences of the surface force especially related to electrical properties of the surface insulation layer of the movable electrode on the $\mathrm{C}-\mathrm{V}$ characteristics

After fabrications, the static electricity can be easily stored in the usual electrostatic microstructures by contacts of their electrode terminals with charged objects. This contact of the electrostatic microstructure with charged bodies was simulated by a circuit shown in Fig. 9. At first, the capacitance $(200 \mathrm{pF})$ was charged by connecting it with a $D C$ voltage source and then the electrode of the device was connected with the charged capacitance by changing a switch circuit. After the electrical charge stored in the device electrodes was discharged, $\mathrm{C}-\mathrm{V}$ characteristics of the device were measured by the circuit of Fig. 7 .

Figure 10 shows changes of the C-V characteristic of a sample device when the charge voltage of the capacitance was changed. It is seen that the hysteresis voltage of the $\mathrm{C}-\mathrm{V}$ curve increases with increase of the charge voltage, i.e. the amount of the electrical charge stored in the condenser. In these case, the surface forees were estimated at $6.0 \mu \mathrm{N}$, $8.4 \mu \mathrm{N}$ and larger than $9.5 \mu \mathrm{N}$ for the charge voltages of $0 \mathrm{~V}$, $20 \mathrm{~V}$ and $40 \mathrm{~V}$, respectively.

The increase of the surface force after the contact with the charged body is thought to come from an accumulation of an electrical charge in the surface $\mathrm{SiO}_{2}$ layer (stopper) or 

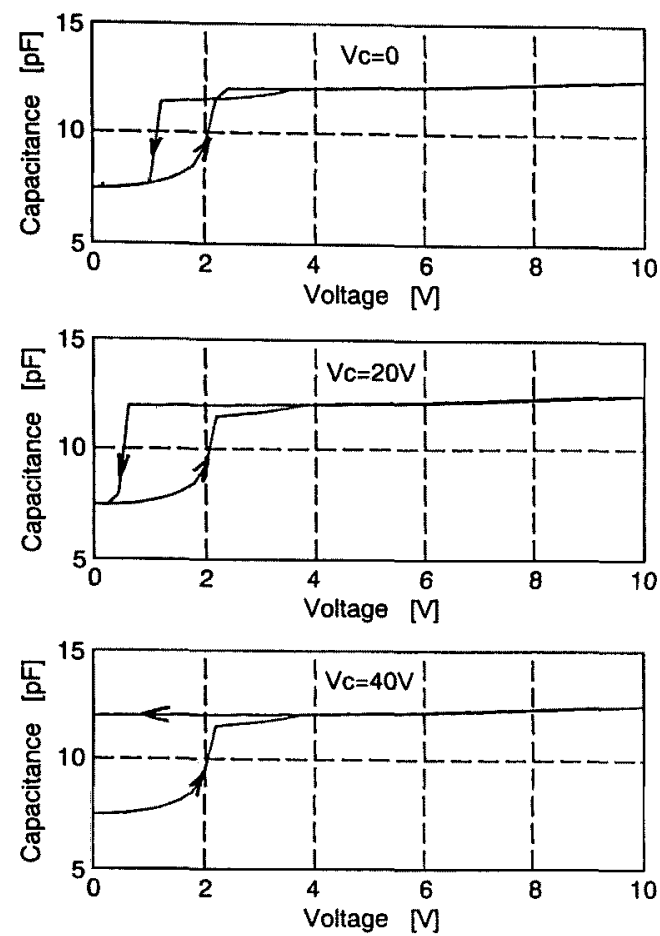

Figure 10: Change of $\mathrm{C}-\mathrm{V}$ characteristics of a sample device when charge voltage of the condenser was changed

a residual polarization of $\mathrm{SiO}_{2}$ layer due to the application of the large electric field to this layer [5].

Figure 11 shows the $\mathrm{C}-\mathrm{V}$ curves of two types of devices with different shapes of surface $\mathrm{SiO}_{2}$ layers. Fig. 11 (a) shows the characteristic of the device shown in Fig. 1, i.e. $\mathrm{SiO}_{2}$ layer $(150 \mu \mathrm{mX} 150 \mu \mathrm{m})$ was formed at the four corners of the upper and lower surfaces of the movable electrode (Sample C). The ratio of the surface oxide area to the total (upper and lower) surface area of the movable electrode was less than $4 \%$. On the other hand, Fig. 11(b) shows the characteristic of the device in which whole of the upper and lower surfaces was covered by $\mathrm{SiO}_{2}$ layer (Sample D). The applied voltage was changed from $0 \mathrm{~V}$ to $+3 \mathrm{~V}$ and decreased to $-3 \mathrm{~V}$ and then increased to $0 \mathrm{~V}$.

The electrostatic force $\mathrm{F}_{e s}$ between two parallel plate electrodes separated by a distance $d$ is given by $F_{e s}=\varepsilon_{0} S^{2} /$ $2 \mathrm{~d}^{2}$ and proportional to square of the applied voltage $V$. This means that the capacitance change between the movable and the fixed electrode is expected to be independent of the polarity of the applied voltage. However, it is observed that the C-V characteristics in Fig. 11 doesn't have symmetry with respect to the axis of $V=0$. The asymmetry of the characteristics seems to come from a shift of a symmetry axis to the positive voltage region. From comparison of the pull-in voltages of

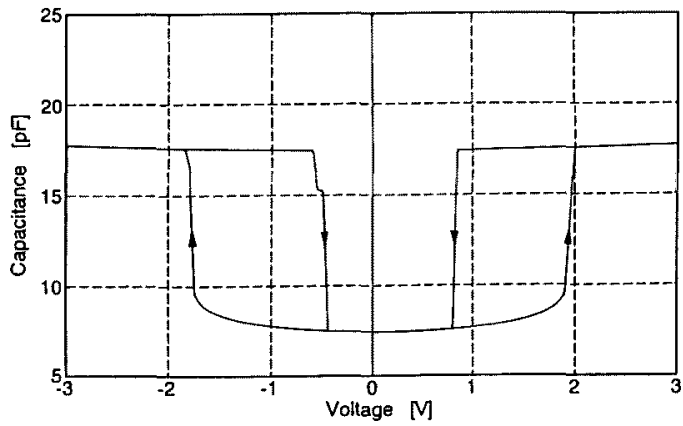

(a)

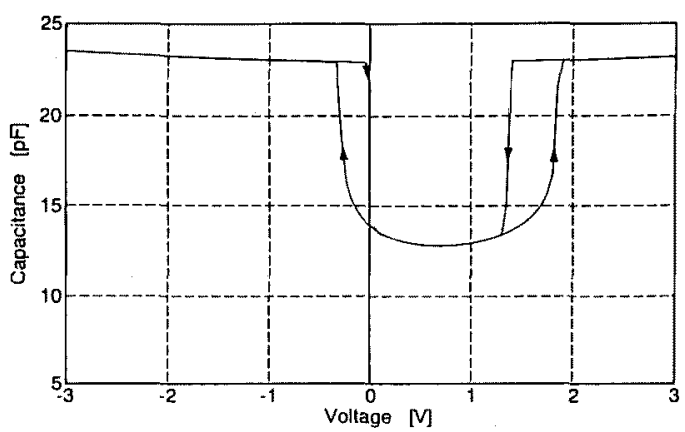

(b)

Figure 11: C-V characteristics of two kinds of samples: (a) $\mathrm{SiO}_{2}$ layer (stopper) is formed at four corner of the upper or lower surface of the movable electrode, (b) whole of the main surfaces of the movable electrode are covered by $\mathrm{SiO}_{2}$ layer

the positive and the negative voltage regions, the shift of the symmetry axis is estimated at $0.1 \mathrm{~V}$ in Fig. 11(a) and from $0.7 \mathrm{~V}$ to $0.8 \mathrm{~V}$ in Fig. 11(b).

In Fig. 11(b), when the voltage was returned to zero just after the application of the minus voltage, the capacitance didn't return to the original value $(13.8 \mathrm{pF})$. This means that the stiction between the movable and the fixed electrodes arose similar to the sample B of Fig. 8 .

One of the possible reasons to cause such a voltage shift (especially in Fig. 11(a)) is an additional bias voltage added to the measurement DC voltage. When two different conductive materials contact each other, a voltage difference (contact potential difference) appears between them. Even if such conductive materials are connected electrically through other conductive materials, the voltage differece of the same magnitude is also generated. In case of the present device, it is thought that the voltage difference which was equal to the contact potential difference of the materials of the movable and the fixed electrodes ( $\mathrm{Si}$ and Mo) appeared between the both electrodes and such a voltage was added to the measurement DC voltage.

On the other hand, the voltage shift was larger in the 
sample having the larger oxide layer (Sample D). This means that the $\mathrm{SiO}_{2}$ layer was related to the shift. As described in the appendix, when a positive charge of an appropriate density $\left(\sim 2 \times 10^{10} / \mathrm{cm}^{2}\right)$ is assumed in the oxide layer, the shift of the $\mathrm{C}-\mathrm{V}$ curve to the positive voltage region and the stiction in Fig. 11(b) can be explained as the effects of this charge.

One of origins of this positive charge is the contact electrification due to the contact of the oxide layer with the fixed electrode. However, the influence of the contant electrification on the $\mathrm{C}-\mathrm{V}$ characteristics and the surface force is thought to be small since the real contact area was much smaller than the observed contact area and the total amount of the generated charge was very little [5].

On the other hand, in the field of semiconductor devices, it is known that various kinds of electrical charges such as mobile ions $\left(\mathrm{Na}^{+}, \mathrm{K}^{+}\right)$and trapped charges exist in the oxidized silicon layer and these charge densities depend on the fabrication process of the device [8]. In $\mathrm{Si}_{-} \mathrm{SiO}_{2} \mathrm{MOS}$ (Metal-Oxide-Semiconductor) devices of the early period (1960's), electrical charge densities in the region of $10^{12} \sim$ $10^{13} / \mathrm{cm}^{2}$ were observed in the $\mathrm{SiO}_{2}$ layer by measurements of $\mathrm{C}-\mathrm{V}$ characteristics of MOS diodes. These charge densities are much larger than the density of electrons $\left(1.8 \times 10^{10} / \mathrm{cm}^{2}\right)$ induced on counter surfaces of a parallel plate condenser when its gap width $\left(D_{0}=3.0 \mu \mathrm{m}\right)$ is the same as that of the present microstructure and the applied voltage is $10 \mathrm{~V}$.

In the electrostatic microstructures, insulation materials including $\mathrm{SiO}_{2}$ are often used to avoid direct electrical contacts of the movable electrode with the fixed one. Therefore, it is necessary to study electro-mechanical effects of the insulation layer on the electrostatic microstructure more precisely.

\section{CONCLUSIONS}

Influences of surface force on a motion of an electrostatic microstructure, which has a movable electrode supported by a cantilever beam and counter fixed electrodes, was studied theoretically and experimentally.

Voltage dependency of the displacement of the movable electrode was analyzed with the energy model which determined the stable displacement state of the movable electrode from the minimum energy state of the total energy of elastic energy, electrostatic energy and adhesion energy between contact surfaces. A hysteresis voltage defined as a difference between a pull-in voltage and a corresponding release voltage increased nonlinearly with increase of surface force in contact parts of the both electrodes. Magnitude range of the surface force in which stiction of the movable electrode occured was derived.

From an experiment which simulated electrical contacts of the device with charged objects, it was seen that the surface force increased with increase of the charge amount of the object.

In measurements of $\mathrm{C}-\mathrm{V}$ characteristics in positive and negatige voltage regions, it was observed that the $\mathrm{C}-\mathrm{V}$ characteristics shifted to the positive voltage region and the shift was larger in a device with larger surface oxide layer of the movable electrode. It is thought that this volage shift was caused by a positive electrical charge remaining in the oxide layer.

\section{APPENDIX}

Effects of an electrical charge in a surface oxide layer on an electrostatic microstructure has been evaluated by a simple theoretical analysis. Figure A-1 shows a model for the study. A surface of a movable electrode is covered by a $\mathrm{SiO}_{2}$ layer (thickness: $\mathfrak{t}$ ). To simplify the consideration, we assume a plane charge (density: $\sigma$ ) as the oxide charge. In Fig. A-1, $x$ is the distance between the plane charge and the $\mathrm{Si} / \mathrm{SiO}_{2}$ interface. A small voltage $\mathrm{V}$ (in which a bending of energy band of $\mathrm{Si}$ is enough small near $\mathrm{Si}_{2} \mathrm{SiO}_{2}$ interface) is applied between the movable electrode and a fixed one. Since electric fields inside of the both electrodes are zero, the next relation can be obtained.

$$
\sigma_{1} / 2 \varepsilon_{0}+\sigma_{2} / 2 \varepsilon_{0}+\sigma / 2 \varepsilon_{0}=0
$$

Where $\sigma_{1}$ and $\sigma_{2}$ are densities of electrical charges induced on the surfaces of the movable electrode and the fixed one, respectively. Electric field $\mathrm{E}_{1}$ between the $\mathrm{Si} / \mathrm{SiO}_{2}$ interface and the oxide charge $\sigma$, electric field $E_{2}$ between the oxide charge $\sigma$ and the $\mathrm{SiO}_{2}$ surface and electric field $\mathrm{E}_{3}$ in the space between the $\mathrm{SiO}_{2}$ layer and the fixed electrode are given by the next equations.

$$
\begin{aligned}
& E_{1}=\left(-\sigma_{1}+\sigma_{2}+\sigma\right) / 2 \varepsilon_{0} \varepsilon_{r} \\
& E_{2}=\left(-\sigma_{1}+\sigma_{2}-\sigma\right) / 2 \varepsilon_{0} \varepsilon_{r} \\
& E_{3}=\left(-\sigma_{1}+\sigma_{2}-\sigma\right) / 2 \varepsilon_{0}
\end{aligned}
$$

Where $\varepsilon_{r}$ is the relative dielectric constant of $\mathrm{SiO}_{2}\left(\varepsilon_{\mathrm{r}}=3.9\right)$. When a voltage difference between the both electrodes is $V$, the relation (A5) is obtained.

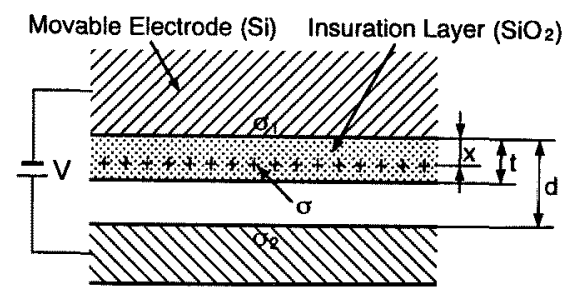

Fixed Electrode (Mo)

Figure A-1: Model for analyzing effects of the oxide charge on the electrostatic microstructure 


$$
E_{1} x+E_{2}(t-x)+E_{3}(d-t)=V
$$

By simultaneously solving eq.(Al) and the equation obtained by substituting eqs.(A2), (A3) and (A4) into eq.(A5), the next surface charge $\sigma_{2}$ is obtained.

$$
\sigma_{2}=\frac{x \sigma-\varepsilon_{0} \varepsilon_{r} V}{\left(\varepsilon_{r}-1\right) t-\varepsilon_{r} d}
$$

The electrostatic force $F_{\text {es }}$ between the movable and the fixed electrodes is given by the next equation.

$$
\begin{aligned}
\mathrm{F}_{\mathrm{es}} & =\left(\sigma_{1}+\sigma\right) \sigma_{2} / 2 \varepsilon_{0} \\
& =-\sigma_{2}^{2} / 2 \varepsilon_{0} \\
& =-\frac{\mathrm{S}}{2 \varepsilon_{0}}\left\{\frac{\mathrm{x} \sigma-\varepsilon_{0} \varepsilon_{\mathrm{r}} \mathrm{V}}{\left(\varepsilon_{\mathrm{r}}-1\right) \mathrm{t}-\varepsilon_{\mathrm{r}} \mathrm{d}}\right\}^{2}
\end{aligned}
$$

From eq.(A7), the voltage $V_{0}$ in which the electrostatic force $F_{e s}$ becomes zero is calcurated as follows.

$$
\mathrm{V}_{0}=\mathrm{x} \sigma / \varepsilon_{0} \varepsilon_{\mathrm{r}}
$$

$\mathrm{V}-\mathrm{F}_{\mathrm{es}}$ curve given by eq.(A7) is symmetry with respect to the axis of $\mathrm{V}=\mathrm{V}_{0}$. Therefore, the voltage $\mathrm{V}_{0}$ becomes the voltage shift of $\mathrm{C}-\mathrm{V}$ curve due to the oxide charge when contact potential difference of the electrode materials is zero.

When the oxide layer contacts with the fixed electrode $(d=t)$ in $V=0$, the electrostatic force $F_{e s}$ of eq.(A7), i.e. the stiction force, becomes as follows.

$$
\mathrm{F}_{\mathrm{cs}}=-\frac{\mathrm{S}}{2 \varepsilon_{0}}\left(\frac{\mathrm{x}}{\mathrm{t}}\right)^{2} \sigma^{2}
$$

For example, when the oxide charge of the density of $\sigma$ $=3.2 \times 10^{-5} \mathrm{C} / \mathrm{m}^{2}\left(2.0 \times 10^{10} / \mathrm{cm}^{2}\right)$ is distributed near the surface of $\mathrm{SiO}_{2}$ layer of thickness of $0.8 \mu \mathrm{m}(\mathrm{x}=0.8 \mu \mathrm{m})$, the voltage shift $\mathrm{V}_{0}$ of the $\mathrm{C}-\mathrm{V}$ characteristic is estimated at $0.74 \mathrm{~V}$ from eq. (A8). Further, the stiction force (surface force) due to this oxide charge between a $\mathrm{SiO}_{2}$ stopper of the size of $150 \mu \mathrm{m} \times 150 \mu \mathrm{m}$ and the fixed electrode is calculated at $1.3 \mu \mathrm{N}$ from eq. (A9).

(Manuscript received Jan. 26, 1998, revised Aug. 10, 1998)

\section{REFERENCES}

[1] S. Suzuki, M. Miki, M. Matsumoto, B. Kloeck, S. Tsuchitani, S. Kuragaki, K. Sato and A. Koide, "Semiconductor Capacitance-type Crash Sensor for Airbag Systems", Proc. of the 3rd Int. Conf. Micro Electro, Opto, Mechanic Systems, pp.383-392, 1992

[2] R. Kaneko, "Microtribology Related to MEMS", Proc. of IEEE Workshop on Micro Electro Mechanical Systems, pp.1-8, 1991

[3] R. Legtenberg, J. Elders and M. Elwenspoek, "Stiction of Surface Micromachined Structures after Rinsing and Drying", Tech. Digest of 7th Int. Conf. Solid-State
Sensors and Actuators, pp.198-201, 1993

[4] S. Tsuchitani, S. Suzuki, S. Shimada, M. Miki, M. Matsumoto and S. Kuragaki, "Measuement of the Surface Force in Micro Structures and Its Reduction (in Japanese)", Trans.of Society of Instrumentation and Control Engineering (SICE), 30, 2, pp.136-142, 1994

[5] S. Tsuchitani, S. Suzuki, S. Shimada, M. Miki, M. Matsumoto and S. Kuragaki, "Surface Force in Electrostatic Type Microsensors and Microactuators (in Japanese)", Trans. of Societyof Instrumentation and Control Engineering (SICE), 30, 2, pp.143-149, 1994

[6] S. Tsuchitani, S. Suzuki, M. Miki and M. Matsumoto, "Theoretical Study on Surface Force in Microstructures (in Japanese)", Trans. of Society of Instrumentation and Control Engineering (SICE), 32, 5, pp.637-645, 1996

[7] T. Ohta, "Strength of Material (in Japanese)", Sankaido, 1975

[8] S. M. Sze, "Physics of Semiconductor Devices", JOHN WILEY \& SONS, 1981

Shigeki Tsuchitani (Member) He received the B.S. and

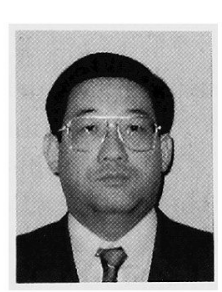
M.S. degrees in material physics from Osaka University in 1977 and 1979, respectively, and the Ph.D degree in electronic physics from Tokyo Institute of Technology in 1994 . Since 1979, he had been working for Hitachi Ltd. and engaged in research and development on sensors. In 1996, he joined the Dep. of Opto-Mechatronics at Wakayama University. His research interests include microsensors, microactuators, their application systems and microphysics.

Reizo Kaneko (Non-Member) He received the B.S., M.S.

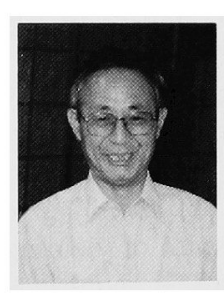
and Ph.D. degrees in mechanical engineering from Kyoto University in 1962, 1964 and 1973, respectively. He joined the NTT Electrical Communications Laboratories in 1964 and since then he had been engaged in research and development on magnetic and optical recording apparatuses and research on surface science and technology including microtribology. Since 1996, he has been a Professor at Wakayama University.

Seiko Suzuki (Member) He received the B.S. degree in

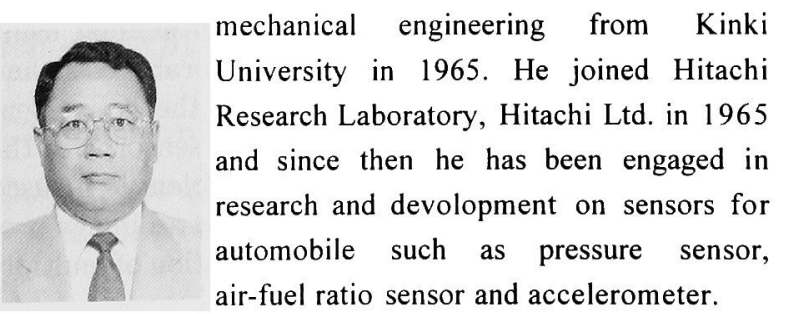

